\title{
On the inverted singlet-triplet gaps and their relevance to organic light-emitting diodes
}

\author{
Piotr de Silva* \\ Department of Energy Conversion and Storage, Technical University of Denmark, \\ Fysikvej 309, 2800 Kongens Lyngby, Denmark \\ E-mail: pdes@dtu.dk
}




\begin{abstract}
The basic design principle for emitters exhibiting thermally activated delayed fluorescence (TADF) is the minimization of the singlet-triplet gap. While typically this gap is positive, a possible inversion of states has been proposed as a pathway to improve the efficiency of organic light-emitting diodes. Despite the efforts to design such emitters, there are very few reports indicating that it is at all possible. We analyze the problem of gap inversion from the perspective of the electronic structure theory. The key result is that inversion is possible but requires a substantial contribution of double excitations and that commonly used cheap electronic structure methods would fail to predict it.
\end{abstract}

\title{
Graphical TOC Entry
}

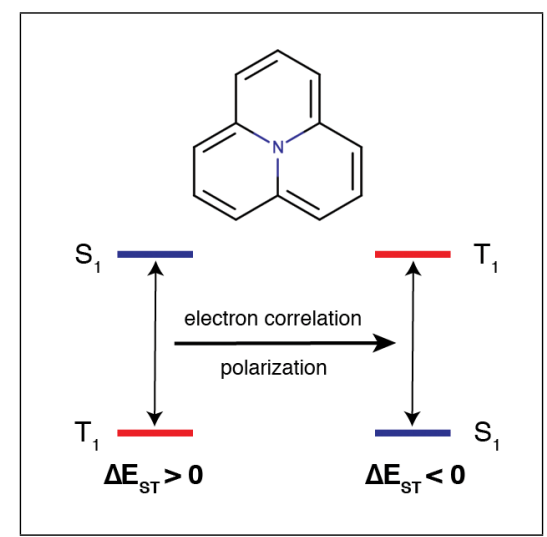


Excited electronic states of molecular systems determine their photophysical and photochemical properties, which are central to many technological and biomedical applications. In particular, investigations of photoactive organic molecules are at the forefront of research due to their potential use in devices such as organic light-emitting diodes (OLEDs), organic photovoltaic cells (OPVs), and molecular sensors. The rational design of new organic emitters or absorbers requires careful engineering of low-lying excited states to optimize the photophysical properties of the molecule for a given application. In the case of OLEDs, the design efforts are focused on maximizing the internal quantum efficiency (IQE) of the emitters by efficient harvesting of triplet excitons, which are normally nonemissive. This has lead to the design of phosphorescent emitters, ${ }^{1,2}$ which contain a heavy-metal atom (e.g. iridium) to enhance the spin-orbit coupling and enable phosphorescence from the lowest-lying triplet state $\left(T_{1}\right)$. More recently, an alternative pathway to the harvesting of triplet excitons has been proposed, which is based on the phenomenon of thermally-activated delayed fluorescence (TADF). ${ }^{3,4}$ In the TADF process, a small gap between the lowest-lying singlet and triplet state $\Delta E_{S T}=E_{S_{1}}-E_{T_{1}}$ enables a thermal up-conversion from the triplet to the singlet manifold and subsequent fluorescence from the bright $S_{1}$ state.

The basic design principle for TADF emitters is to make molecules composed of donor and acceptor units with spatially separated HOMO and LUMO orbitals, which give rise to excited states with predominantly charge-transfer $(\mathrm{CT})$ character. $^{5-7}$ The CT states are characterized by small exchange couplings, which results in small $\Delta E_{S T}$ values allowing for a significant equilibrium population in the $S_{1}$ state. While a pure CT character of the $S_{1}$ and $T_{1}$ states would preclude efficient reverse intersystem crossing and fluorescence needed for the overall high TADF rate, it has been realized that a low-lying local excitation (LE) also plays a role in the TADF mechanism. ${ }^{8-11}$ Recently de Silva et al. proposed a fourstate model ${ }^{12}$ explaining the possibility of efficient TADF resulting from mixing of diabatic $\mathrm{CT}$ and LE states to form mixed-character adiabatic states enabling simultaneously small $\Delta E_{S T}$, and relatively high spin-orbit coupling and oscillator strength. The sampling of the 
parameter space of the model gave solutions with negative singlet-triplet gaps, which could suggest that such realizations are possible in real molecular systems.

For the vast majority of molecular systems, the $T_{1}$ state lies energetically below the $S_{1}$ state. This is due to exchange interactions, which stabilize triplets and destabilize singlets leading to a positive $\Delta E_{S T}$. However, there is a growing interest in designing molecules with inverted states, which would allow replacing the up-conversion in TADF with a more efficient down-conversion. ${ }^{13-17}$ Several mechanisms have been identified as a possible path to overcome the exchange interactions and lead to negative singlet-triplet gaps. Difley et al. found through constrained density functional theory calculations that state inversion is possible in exciplexes. ${ }^{13}$ It was explained by the kinetic exchange mechanism, which stabilizes singlet states. Olivier et al. found that the presence of a polarizable environment also leads to stabilization of singlets and enables negative $\Delta E_{S T} \cdot{ }^{18}$ Eizner et al. found that placing a molecule in a microcavity can give inverted polaritonic states. ${ }^{14}$ Another possibility for effectively inverted states is to inhibit internal conversion in the triplet manifold so that $T_{2}$ lying above $S_{1}$ is the lowest populated triplet state. ${ }^{15}$ Despite these efforts, an indisputable ab initio prediction of a molecular system with inverted singlet and triplet states is missing. This relates to the fact that it is not understood what is required from the electronic structure of a molecule to exhibit such inversion. Gaining such understanding would possibly enable the rational design of emitters with negative $\Delta E_{S T}$ and lead to OLED devices with improved efficiency. In this Letter, we show how the presence of low-lying states of a doubly excited character is a prerequisite for the singlet-triplet gap inversion and rationalize the prior findings based on the kinetic exchange mechanism and interaction with a polarizable environment. We also give an example of a molecule that exhibits such an inversion, which is confirmed with ab initio calculations.

The simplest ab initio electronic structure theory that can describe excited states is configuration interaction singles (CIS). The CIS Hamiltonian is given in the basis of singlyexcited configurations (Slater determinants) $\left\{\left|\Psi_{i}^{a}\right\rangle\right\}$, which are generated by promoting an 
electron from an orbital $i$ occupied in the reference determinant to an empty orbital $a$. The singly-excited basis states are of the spin-mixed character, but their linear combinations can be formed to generate spin-pure configuration state functions (CSFs):

$$
\begin{aligned}
\left|{ }^{1} \Phi_{i}^{a}\right\rangle & =\frac{1}{\sqrt{2}}\left(\left|\Psi_{i}^{a}\right\rangle+\left|\Psi_{\bar{i}}^{\bar{a}}\right\rangle\right) \quad \text { (singlet) } \\
\left|{ }^{3} \Phi_{i}^{a}\right\rangle & =\frac{1}{\sqrt{2}}\left(\left|\Psi_{i}^{a}\right\rangle-\left|\Psi_{\bar{i}}^{\bar{a}}\right\rangle\right) \quad \text { (triplet) }
\end{aligned}
$$

where the bar over a spin-orbital index denotes the $\beta$ spin and lack thereof denotes the $\alpha$ spin. Using the CSF basis in eq. 1 and the Brillouin's theorem, which says that the ground-state does not couple with singly-excited configurations, it is possible to split the total Hamiltonian into a direct sum of Hamiltonians operating on the ground state, and in the excited singlet and triplet subspaces $\mathbf{H}=\mathbf{H}_{\mathbf{0}} \oplus{ }^{1} \mathbf{H} \oplus{ }^{3} \mathbf{H}$. The latter two Hamiltonians can be directly diagonalized to give energies and eigenvectors of the excited states. In the following we will show that the CIS Hamiltonian can never give a negative singlet-triplet gap. First, we will make use of a useful inequality in linear algebra, ${ }^{19}$

Weyl's inequality If $\mathbf{A}$ and $\mathbf{B}$ are Hermitian matrices with eigenvalues $a_{1} \geq a_{2} \geq \ldots \geq a_{n}$ and $b_{1} \geq b_{2} \geq \ldots \geq b_{n}$, and $\mathbf{C}=\mathbf{A}+\mathbf{B}$ with eigenvalues $c_{1} \geq c_{2} \geq \ldots \geq c_{n}$, then for $i=1,2, \ldots, n$

$$
a_{i}+b_{n} \leq c_{i} \leq a_{i}+b_{1}
$$

In particular, the lowest eigenvalue of $\mathbf{C}$ is bounded from both sides

$$
a_{n}+b_{n} \leq c_{n} \leq a_{n}+b_{1}
$$

Considering eq. 3 and that $\lambda_{n}\left({ }^{3} \mathbf{H}\right)=-\lambda_{1}\left(-{ }^{3} \mathbf{H}\right)$, the singlet-triplet gap is bounded from below by the lowest eigenvalue of the difference between the singlet and triplet Hamiltonians:

$$
\Delta E_{S T}=E_{S_{1}}-E_{T_{1}}=\lambda_{n}\left({ }^{1} \mathbf{H}\right)+\lambda_{1}\left(-{ }^{3} \mathbf{H}\right) \geq \lambda_{n}\left({ }^{1} \mathbf{H}-{ }^{3} \mathbf{H}\right)
$$


By using Slater-Condon rules it is straightforward to show that that the elements of ${ }^{1} \mathbf{H}-{ }^{3} \mathbf{H}$ in the CSF basis are

$$
\left\langle{ }^{1} \Phi_{i}^{a}|\mathbf{H}|^{1} \Phi_{j}^{b}\right\rangle-\left\langle{ }^{3} \Phi_{i}^{a}|\mathbf{H}|^{3} \Phi_{j}^{b}\right\rangle=2\left\langle\Psi_{i}^{a}|\mathbf{H}| \Psi_{\bar{j}}^{\bar{b}}\right\rangle=2(i a|| \bar{j} \bar{b})=2(i a \mid j b)
$$

where the latter two objects are the antisymmetrized and standard two-electron Coulomb integrals in the chemists' notation. Since ${ }^{1} \mathbf{H}-{ }^{3} \mathbf{H}$ is simply twice the matrix of two-electron integrals, which is known to be positive semidefinite, ${ }^{20}$ its lowest eigenvalue $\lambda_{n}\left({ }^{1} \mathbf{H}-{ }^{3} \mathbf{H}\right) \geq 0$ and $\Delta E_{S T} \geq 0$ for any CIS Hamiltonian.

This result carries over also to the time-dependent Hartree-Fock (TDHF) method, which solves a non-Hermitian eigenvalue problem to obtain the excitation energies $\omega$ from the Hartree-Fock ground state

$$
\left(\begin{array}{cc}
\mathbf{A} & \mathbf{B} \\
-\mathbf{A}^{*} & -\mathbf{B}^{*}
\end{array}\right)\left(\begin{array}{l}
\mathbf{X} \\
\mathbf{Y}
\end{array}\right)=\omega\left(\begin{array}{l}
\mathbf{X} \\
\mathbf{Y}
\end{array}\right)
$$

where $A_{i a, j b}=\delta_{i j} \delta_{a b}\left(\epsilon_{a}-\epsilon_{i}\right)+(i a \| j b)$ and $B_{i a, j b}=(i a \| b j)$. The matrix in eq. 6 can be block-diagonalized to decouple singlet and triplet excitations giving the following blocks of the matrices: ${ }^{1} A_{i a, j b}=A_{i a, j b}+A_{i a, \bar{j} \bar{b}},{ }^{1} B_{i a, j b}=B_{i a, j b}+B_{i a, \bar{j} \bar{b}}$ for the singlets, and ${ }^{3} A_{i a, j b}=$ $A_{i a, j b}-A_{i a, \bar{j} \bar{b}},{ }^{3} B_{i a, j b}=B_{i a, j b}-B_{i a, \bar{j} \bar{b}}$ for the triplets. While the problem remains nonHermitian, which prevents direct application of the Weyl's inequality to provide a lower bound on the singlet-triplet gap, the equations can be reduced to Hermitian eigenvalue problems for the squares of excitation energies

$$
\left({ }^{\Sigma} \mathbf{A}-{ }^{\Sigma} \mathbf{B}\right){ }^{1 / 2}\left({ }^{\Sigma} \mathbf{A}+{ }^{\Sigma} \mathbf{B}\right)\left({ }^{\Sigma} \mathbf{A}-{ }^{\Sigma} \mathbf{B}\right){ }^{1 / 2}\left({ }^{\Sigma} \mathbf{Z}\right)={ }^{\Sigma} \omega^{2}\left({ }^{\Sigma} \mathbf{Z}\right)
$$

where ${ }^{\Sigma} \mathbf{Z}=\left({ }^{\Sigma} \mathbf{A}-{ }^{\Sigma} \mathbf{B}\right){ }^{1 / 2}\left({ }^{\Sigma} \mathbf{X}+{ }^{\Sigma} \mathbf{Y}\right)$, and $\Sigma=\{1,3\}$. It can be shown that $\mathbf{C}=\left({ }^{1} \mathbf{A}-{ }^{1} \mathbf{B}\right)=$ $\left({ }^{3} \mathbf{A}-{ }^{3} \mathbf{B}\right)=\delta_{i j} \delta_{a b}\left(\epsilon_{a}-\epsilon_{i}\right)+(i b \mid a j)-(i j \mid a b)$, and the difference between the singlet and 
triplet operators becomes

$$
\mathbf{C}^{1 / 2}\left[{ }^{1} \mathbf{A}+{ }^{1} \mathbf{B}-{ }^{3} \mathbf{A}-{ }^{3} \mathbf{B}\right] \mathbf{C}^{1 / 2}
$$

with

$$
\left[{ }^{1} \mathbf{A}+{ }^{1} \mathbf{B}-{ }^{3} \mathbf{A}-{ }^{3} \mathbf{B}\right]=4(i a \mid j b)
$$

Since it is again a positive semidefinite matrix of two-electron integrals, and $\mathbf{C}$ has to be positive semidefinite for the square root to exist, the entire difference matrix in eq. 8 is positive semidefinite. Using again the Weyl's inequality we obtain $\omega_{S_{1}}^{2}-\omega_{T_{1}}^{2}=\left(\omega_{S_{1}}-\right.$ $\left.\omega_{T_{1}}\right)\left(\omega_{S_{1}}+\omega_{T_{1}}\right) \geq 0$, which directly implies that $\Delta E_{S T} \geq 0$ for any TDHF calculation. Since both CIS and TDHF are uncorrelated methods, these results suggest that if inverted gaps are possible at all, they would emerge as an effect of electron correlation.

To put this result in the context of TADF emitters and illustrate the effect of going beyond single excitations, we turn to the recently proposed four-state model of TADF. ${ }^{12}$ The model Hamiltonian is given in the basis of spin-mixed singly-excited configurations within a 3 -orbital active space $\left\{\phi_{A}, \phi_{B}, \phi_{C}\right\}$. The $4 \times 4$ matrix can be block-diagonalized to form respective singlet and triplet Hamiltonians, and their difference is

$$
{ }^{1} \mathbf{H}-{ }^{3} \mathbf{H}=2\left(\begin{array}{cc}
K_{C T} & K_{X} \\
K_{X} & K_{L E}
\end{array}\right)
$$

with the matrix elements given by two-electron integrals $K_{C T}=\left(\phi_{A} \phi_{B} \mid \phi_{B} \phi_{A}\right), K_{L E}=$ $\left(\phi_{A} \phi_{C} \mid \phi_{C} \phi_{A}\right)$, and $K_{X}=\left(\phi_{A} \phi_{C} \mid \phi_{B} \phi_{A}\right)$. The positive semidefinitness of this matrix implies that $K_{X}^{2} \leq K_{C T} K_{L E}$, which is the well-know Cauchy-Schwarz inequality used in the electron integral prescreening, and that $\Delta E_{S T} \geq 0$.

It is clear that since the four-state Hamiltonian is of the CIS type, the singlet-triplet gap is strictly non-negative. To understand the effect of double excitations on the magnitude and sign of the gap, we consider a minimal three-state model where the basis states are 
two spin-mixed CT configurations $\{|C T 1\rangle,|C T 2\rangle\}\left(\varphi_{A} \rightarrow \varphi_{B}\right.$ transitions $)$ and a doublyexcited configuration $|D\rangle\left(\left(\varphi_{A}\right)^{2} \rightarrow\left(\varphi_{B}\right)^{2}\right.$ transition $)$. The Hamiltonian in the basis of these configurations takes the following form

$$
\mathbf{H}=\left(\begin{array}{ccc}
0 & K_{C T} & t_{C T, D} \\
K_{C T} & 0 & t_{C T, D} \\
t_{C T, D} & t_{C T, D} & E_{D}
\end{array}\right)
$$

where $E_{D}$ is the energy gap between $|C T\rangle$ and $|D\rangle$ configurations, $K_{C T}$ if the exchange integral, and the coupling between $|C T\rangle$ and $|D\rangle$ states is a sum of one- and two-electron integrals $t_{C T, D}=h_{A B}+\left(\varphi_{A} \varphi_{B} \mid \varphi_{B} \varphi_{B}\right)$. In the basis of spin-pure CSFs, the corresponding singlet Hamiltonian and the energy of the only triplet state are

$$
{ }^{1} \mathbf{H}=\left(\begin{array}{cc}
K_{C T} & \sqrt{2} t_{C T, D} \\
\sqrt{2} t_{C T, D} & K_{C T}
\end{array}\right), \quad E_{T}=-K_{C T}
$$

By subtracting the triplet energy from the diagonal of the singlet Hamiltonian and using the Weyl's inequality (eq. 3), one obtains a condition for the negative lower bound on the singlet-triplet gap

$$
t_{C T, D}^{2}>K_{C T}\left(E_{D}+K_{C T}\right)
$$

Since doubly-excited configurations $\left|\Psi_{i \bar{i}}^{a \bar{a}}\right\rangle$ can mix only with CSFs of singlet multiplicity, adding them leads to the reduction of the singlet-triplet gap. If the coupling is strong (large $\left.t_{C T, D}^{2}\right)$ and/or singly- and doubly-excited configurations are energetically close (small $E_{D}$ ), a substantial admixture of the double excitation in the $S_{1}$ state can lead to an inverted singlettriplet gap, which would be a manifestation of the electron correlation effect in the excited state. This is effectively the same mechanism as the kinetic exchange, which is explained as a two-step process where the electrons are swapped by pairing them first in one orbital. The singlet-triplet gap predicted by the kinetic exchange model is $\Delta E_{S T}=K-\frac{t^{2}}{\Delta E}$, where $K$ is 
the direct exchange coupling, $t$ is the hopping integral between involved orbitals, and $\Delta E$ is the energy difference between the paired and unpaired states. ${ }^{13}$ To obtain this result from the model Hamiltonian in eq. 12 one can use the downfolding technique ${ }^{21}$ to integrate out the doubly-excited state. The resolvent for the Hamiltonian in eq. 12 is

$$
\mathbf{G}(\omega)=\left(\omega-{ }^{1} \mathbf{H}\right)^{-1}=\left(\begin{array}{cc}
\omega-K_{C T} & \sqrt{2} t_{C T, D} \\
\sqrt{2} t_{C T, D} & \omega-K_{C T}
\end{array}\right)^{-1}
$$

Carrying out the inversion explicitly, one obtains

$$
\mathbf{G}_{00}(\omega)=\left(\omega-\left[K_{C T}+2 t_{C T, D}^{2}\left(\omega-E_{D}\right)^{-1}\right]\right)^{-1}
$$

which is a resolvent for a one-dimensional energy-dependent effective Hamiltonian ${ }^{1} \mathbf{H}_{e f f}(\omega)=$ $2 t_{C T, D}^{2}\left(\omega-E_{D}\right)^{-1}$. If the coupling to the double excitation is small and/or the energy gap is large, the effective Hamiltonian can be approximated with an energy-independent one by replacing $\omega$ with the energy of the singly-excited configuration $\left({ }^{1} \mathbf{H}_{00}=K_{C T}\right)$

$$
{ }^{1} \tilde{\mathbf{H}}_{e f f}={ }^{1} \mathbf{H}_{e f f}\left(\omega=K_{C T}\right)=K_{C T}+2 t_{C T, D}^{2}\left(K_{C T}-E_{D}\right)^{-1}
$$

This leads to the expression for the energy of the singlet state $E_{S_{1}}=K_{C T}-2 \frac{t_{C T, D}^{2}}{E_{D}-K_{C T}}$, and the gap $\Delta E_{S T}=2\left(K_{C T}-\frac{t_{C T, D}^{2}}{E_{D}-K_{C T}}\right)$. This is essentially the same results as in the kinetic exchange model, except that $t_{C T, D}$ is now not only a hopping integral but also contains a two-electron $\left(\varphi_{A} \varphi_{B} \mid \varphi_{B} \varphi_{B}\right)$ term.

The results presented above clearly show that i) to realize a molecular system with a negative singlet-triplet gap, the $S_{1}$ state has to be to some extent of a doubly-excited character, and ii) if such system was found, uncorrelated methods would still incorrectly predict that the gap is positive. Since the vast majority of excitation energy calculations are done at the level of the linear-response time-dependent density functional theory (TD-DFT), 
it is of interest to discuss if negative gaps can be predicted within this framework. On the one hand TD-DFT accounts for electron correlation through the exchange-correlation kernel $\left(f_{x c}\left(\mathbf{r}, \mathbf{r}^{\prime}, \omega\right)\right)$, but on the other it is well known that it fails to describe double excitations if a frequency-independent kernel is used (adiabatic approximation). ${ }^{22}$ Within the TammDancoff approximation, which corresponds directly to the CIS method, ${ }^{23}$ the excitation energies are eigenvalues of the matrix equation $\mathbf{A X}=\omega \mathbf{X}$, where the matrix elements $A_{i a, j b}=\delta_{i j} \delta_{a b}\left(\epsilon_{a}-\epsilon_{i}\right)+(i a \mid j b)+\left(i a\left|f_{x c}\right| j b\right)$. Working again in the CSF basis, the difference between the corresponding singlet and triplet operators is

$$
\left\langle{ }^{1} \Phi_{i}^{a}|\mathbf{A}|^{1} \Phi_{j}^{b}\right\rangle-\left\langle{ }^{3} \Phi_{i}^{a}|\mathbf{A}|^{3} \Phi_{j}^{b}\right\rangle=2(i a \mid j b)+2\left(i a\left|f_{x c}^{\alpha, \beta}\left(\mathbf{r}, \mathbf{r}^{\prime}, \omega\right)\right| j b\right) .
$$

In contrast with the CIS case, now the definiteness of this matrix depends on the properties of the exchange-correlation kernel. In practice, TD-DFT calculations are typically done in the adiabatic approximation, where the kernel is frequency-independent. Even so, the adiabatic kernel $f_{x c}^{\alpha, \beta}\left(\mathbf{r}, \mathbf{r}^{\prime}\right)$ is negative definite, so the matrix in eq. 17 is in general indefinite. Therefore, negative singlet-triplet gaps could in principle occur in TD-DFT calculations. However, for closed-shell ground states, it is only the correlation part that contributes to $f_{x c}^{\alpha, \beta}\left(\mathbf{r}, \mathbf{r}^{\prime}\right)$. The correlation contribution, at least for common approximations, is very small compared to the Coulomb interaction ${ }^{24}$ and the matrix in eq. 17 turns out to be positive definite in practice and $\Delta E_{S T} \geq 0$ should be expected from such calculations. Nevertheless, the negative definiteness of the xc kernel contribution clearly shows that electron correlation leads to the reduction of the singlet-triplet gap. Eventually, for sufficiently correlated excited states this may lead to the gap inversion. For such systems, the adiabatic approximation breaks down and frequency-dependent kernels are needed to account for the mixing with doubly-excited configurations.

The discussion above shows that electron correlation effects, in particular contribution of double excitations in the $S_{1}$ state, are a prerequisite for a negative singlet-triplet gap. 
This situation is realized at conical intersections (CI) between $S 1$ and $S 0$ states, where $T 1$ naturally lies above both singlets. Of course, this observation is useless from the perspective of the design of organic emitters as accessible CIs inevitably lead to radiationless relaxation. Nevertheless, it again highlights the importance of double excitations which are known to be crucial for the description of CIs. Therefore, all the electronic structure methods that fail at describing conical intersections, including CIS and adiabatic TD-DFT, are bound to fail at predicting any possible singlet-triplet gap inversions. For such cases one needs to resort to wavefunction-based methods that include doubles explicitly, use TD-DFT with a frequency-dependent kernel like Dressed TD-DFT, ${ }^{25,26}$ or other practical DFT approaches that allow for explicit or implicit inclusion of double excitations like Spin-Flip TD-DFT ${ }^{27}$ or Constrained DFT, ${ }^{28}$ which have been shown to be able to produce conical intersections. ${ }^{29-31}$

In the context of OLEDs exhibiting TADF, it has been realized that also the presence of a polarizable medium can significantly affect the singlet-triplet gap. ${ }^{32,33}$ In a typical scenario, the $S_{1}$ state has more CT character than the $T_{1}$ state; ${ }^{11}$ therefore, it is stabilized stronger leading to smaller $\Delta E_{S T}$. Olivier et al. have found that a combination of TD-DFT calculations with polarizable microelectrostatic embedding could indeed lead to negative gaps. ${ }^{17,18}$ In light of the findings in this Letter, it becomes unclear whether this result is physical or is just an artifact of the classical and approximate treatment of the environment. In the end, full TD-DFT calculations for the entire molecule + environment system would most likely give a positive gap. More broadly, it is an interesting question to ask if interactions with the environment can ever lead to negative gaps. In this context, it is interesting to notice that Subotnik showed that orbital relaxation in the excited state has the same effect on energies of CT states as adding double excitations. ${ }^{34,35}$ This can be also rationalized for the interaction between the emitter and its environment. Let us assume that the exchange interactions between the subsystems are negligible and that the mean-field ground-state wavefunction of the entire molecule+environment $(\mathrm{M}+\mathrm{E})$ system can be factorized into a product of the subsystem wavefunctions $\left|\Psi_{0}^{M+E}\right\rangle=\left|\Psi_{0}^{M}\right\rangle\left|\Psi_{0}^{E}\right\rangle$. An excited state of the emitter within the 
CIS and frozen environment approximations takes the following form

$$
\left|\Psi_{n}^{M+E}\right\rangle=\left(\left|\Psi_{0}^{M}\right\rangle+\sum_{i a} t_{i}^{a} a_{a}^{\dagger} a_{i}\left|\Psi_{0}^{M}\right\rangle\right)\left|\Psi_{0}^{E}\right\rangle
$$

where we use the intermediate normalization of the wavefunctions. After the excitation in the subsystem $\mathrm{M}$, the environment polarizes its electronic degrees of freedom, which can be described as relaxing the orbitals and adopting a different mean-field wavefunction $\left|\Psi_{0, p o l}^{E}\right\rangle$, which is not orthogonal to the frozen one $\left(\left\langle\Psi_{0}^{E} \mid \Psi_{0, p o l}^{E}\right\rangle \neq 0\right)$. According to the Thouless theorem, ${ }^{36}$ we can connect these two determinants by using single excitations as the generator $\left|\Psi_{0, p o l}^{E}\right\rangle=\exp \left(\sum_{j b} t_{j}^{b} t_{j}^{b} a_{b}^{\dagger} a_{j}\right)\left|\Psi_{0}^{E}\right\rangle$. By truncating the exponential operator at the single excitations level we obtain the wavefunction of an excited emitter in the polarized environment in the following form

$$
\begin{aligned}
\left|\Psi_{n}^{M+E}\right\rangle & =\left(\left|\Psi_{0}^{M}\right\rangle+\sum_{i a} t_{i}^{a} a_{a}^{\dagger} a_{i}\left|\Psi_{0}^{M}\right\rangle\right)\left(\left(\left|\Psi_{0}^{E}\right\rangle+\sum_{j b} t_{j}^{b} a_{b}^{\dagger} a_{j}\left|\Psi_{0}^{E}\right\rangle\right)\right. \\
& =\left(1+\sum_{i a} t_{i}^{a} a_{a}^{\dagger} a_{i}+\sum_{j b} t_{j}^{b} a_{b}^{\dagger} a_{j}+\sum_{i j a b} t_{i}^{a} t_{j}^{b} a_{a}^{\dagger} a_{b}^{\dagger} a_{i} a_{j}\right)\left|\Psi_{0}^{M+E}\right\rangle,
\end{aligned}
$$

where $\{i, a\}$ run over orbitals in $\left|\Psi_{0}^{M}\right\rangle$, and $\{j, b\}$ in $\left|\Psi_{0}^{E}\right\rangle$. Now it becomes evident how selected doubly-excited configurations are necessary to describe polarization of the environment's density after an excitation of the emitter. Now let $\left|\Psi_{i j}^{a b}\right\rangle$ denote a doubly-excited configuration where one electron is excited in the embedded subsystem $(i \rightarrow a)$ and the other in the environment $(j \rightarrow b)$. The coupling between the doubly- and singly-excited configuration is $\left\langle\Psi_{i j}^{a j}|\mathbf{H}| \Psi_{i j}^{a b}\right\rangle=h_{j b}+\sum_{k \in\left|\Psi_{i j}^{a j}\right\rangle}(j b|| k k)$. Since $\left|\Psi_{0}^{M+E}\right\rangle$ is defined as the Hartree-Fock state which diagonalizes the Fock matrix, the off-diagonal element $F_{j b}=h_{j b}+\sum_{k \in\left|\Psi_{0}^{M+E}\right\rangle}(j b|| k k)$ is zero . Therefore, inserting $h_{j b}=-\sum_{k \in\left|\Psi_{0}^{M+E}\right\rangle}(j b|| k k)$ into the preceding equation yields

$$
\begin{aligned}
\left\langle\Psi_{i j}^{a j}|\mathbf{H}| \Psi_{i j}^{a b}\right\rangle & =\sum_{k \in\left|\Psi_{i j}^{a j}\right\rangle}(j b|| k k)-\sum_{k \in\left|\Psi_{0}^{M+E}\right\rangle}(j b|| k k)=(j b|| a a)-(j b|| i i) \\
& =[(j b \mid a a)-(j b \mid i i)]+[(j i \mid i b)-(j a \mid i b)] .
\end{aligned}
$$


The second term in the final line of the equation above vanishes as we have assumed negligible exchange interactions between the subsystem and the environment, while the first term is the Coulomb repulsion between a charge density localized in the environment and a difference of densities of orbitals $a$ and $i$. If $(i \rightarrow a)$ is a local excitation, the orbital densities would be very similar and mostly cancel each other; therefore, LE states do not couple strongly with the considered double excitations. On the contrary, for a CT excitation the coupling becomes significant as the orbital densities are localized in different parts of the molecule by definition. Since CT configurations are coupled with double excitations stronger than the local excitations, either adding doubles or accounting for classical charge polarization leads to their stabilization, where both effects have the same physical meaning. Therefore, polarizable embedding schemes effectively mimic the effect of including double excitations in the wavefunction of the entire system. Considering that the $S_{1}$ state of TADF emitters has more $\mathrm{CT}$ character than $T_{1}$ and that, as we have shown, double excitations lead to the reduction of singlet-triplet gaps, the negative gaps predicted by polarizable embedding calculations can be physical. More generally, the conclusion is that designing molecules with intrinsically small gaps and embedding them in a polarizable environment, could effectively lead to a gap inversion as have been argued by others.

To illustrate the analytical results with ab initio calculations we have chosen cycl[3.3.3]azine as a model system (fig. 1a). It was speculated that this molecule has a negative singlet-triplet gap, ${ }^{37}$ while some experiments suggest that the gap is very small but positive. ${ }^{38}$ The tiny (or potentially negative) gap of cycl[3.3.3]azine has been attributed to the disjoint character of its HOMO and LUMO, where both frontier orbitals are peaked in different spatial regions (see fig. 1b,c). The resulting small exchange HOMO-LUMO integral can explain the small singlet-triplet splitting in the one-electron HOMO to LUMO excitation picture. To evaluate the effect of electron correlation, we have calculated the lowest singlet and triplet excitation energies with a range of excited-state methods (Table 1).

Both uncorrelated methods (CIS and TD-HF) predict the gap to be positive. This is 
a)

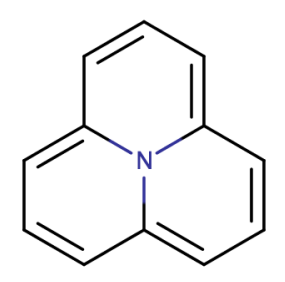

b)

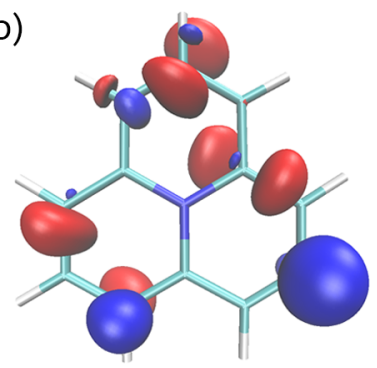

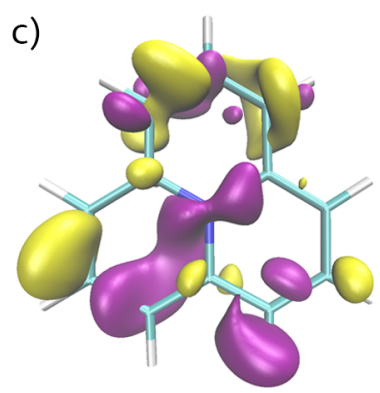

Figure 1: Structure (a), HOMO (b), and LUMO (c) of cycl[3.3.3]azine. HOMO and LUMO calculated at the Hartree-Fock level.

Table 1: Singlet and triplet vertical excitation energies $[\mathrm{eV}]$ calculated with different excited-state electronic structure methods and the resulting singlettriplet gaps.

\begin{tabular}{lccc}
\hline Method & $E_{S_{1}}$ & $E_{T_{1}}$ & $\Delta E_{S T}$ \\
\hline CIS & 1.83 & 1.50 & +0.33 \\
TD-HF & 1.68 & 1.08 & +0.60 \\
PBE & 1.21 & 1.02 & +0.19 \\
B3LYP & 1.26 & 1.05 & +0.21 \\
M06-2X & 1.27 & 1.08 & +0.19 \\
CIS(D) & 1.07 & 1.37 & -0.30 \\
ADC(2) & 1.04 & 1.20 & -0.16 \\
EOM-CCSD & 1.09 & 1.19 & -0.10 \\
\hline
\end{tabular}


also the case for TD-DFT with three different exchange-correlation functionals; the gaps are reduced but the adiabatic correlation kernels are not able to push the singlet state below the triplet. The situation changes dramatically when doubly-excited configurations are treated explicitly in any form. All three correlated wavefunction-based methods predict the gap to be negative, which clearly demonstrates that the molecules with the inverted singlet-triplet structure are possible. This result is promising from the perspective of the design of OLED materials, as it shows that the TADF mechanism can be made even more efficient by down-converting triplet excitons to singlets instead of a thermally-activated upconversion. Actual design of such emitters is challenging not only because molecules with inverted singlet-triplet gaps are exceedingly rare, but also because the $S_{1}$ state is not very bright in these cases. This is indeed what happens for cycl[3.3.3]azine, which is known to violate the Kasha's rule and have a stronger emission from the $S_{2}$ state. ${ }^{38}$ The quenching of the $S_{1}$ state has been attributed to a thermally accessible $S 0-S 1$ conical intersection. This is consistent with the fact that the singlet-triplet inversion requires some contribution of a double excitation, and doubly-excited states are normally dark states and often associated with the presence of conical intersections. Therefore, a potential OLED emitter with a gap inversion should have an $S_{1}$ state which is to some extent of a CT character to minimize exchange interactions, doubly-excited character to further stabilize the singlet state, and locally-excited character to afford appreciable oscillator strength. Perhaps such emitters could be found either by ingenious design or sheer serendipity, this is to be seen. In any case, strong vibronic coupling could help to realize these contrasting requirements, as it is already the case for TADF emitters.

In summary, we have shown both analytically and numerically that electron correlation in the form of double excitations always leads to the reduction of the singlet-triplet gap in molecular systems. In particular, strong coupling with doubles, or strong excited-state electron correlation in general, can generate the inversion of $S_{1}$ and $T_{1}$ states, where the former becomes an energetically lower state. These findings are relevant for the design of 
new OLED emitters based on the TADF mechanism, where minimization of $\Delta E_{S T}$ is the basic design principle. In this work, we argue that the design of emitters with inverted gaps is in principle possible, but requires either assuring that the emitting CT state has a substantial contribution of its doubly-excited configurations or that it couples strongly to the polarizable environment. While the latter strategy has been already pursued in the design of OLED materials, the former is a new result that may lead to a completely new class of molecular emitters. The analysis of popular electronic structure methods for excited states shows that extra care needs to be taken when attempting to computationally predict negative singlet-triplet gaps. We prove that gap inversion is not possible to obtain with uncorrelated excited-state methods and argue that it is highly unlikely with the linear response TD-DFT in the adiabatic approximation. Therefore, to study systems with possible inverted gaps one has to resort to methods capable of describing excited states with relatively strong electron correlation effects.

\section{Computational methods}

All electronic structure calculations were done using the Q-Chem 5.0 package. ${ }^{39}$ The groundstate geometry was optimized at the DFT/B3LYP level. Vertical singlet and triplet excitation energies were calculated using wavefunction methods: CIS, TD-HF, CIS(D),${ }^{40}$ $\mathrm{ADC}(2),{ }^{41}$ EOM-CCSD ${ }^{42}$ and linear response TD-DFT with PBE, B3LYP, and M06-2X exchange-correlation functionals. The cc-pVDZ basis set was used for all the calculations, and the rimp2-cc-pVDZ auxiliary basis set was used for the methods using the resolutionof-identity approximation.

\section{Acknowledgement}

I thank Troy Van Voorhis for helpful comments and encouragement to work on this problem. 


\section{References}

(1) Adachi, C.; Baldo, M. A.; Thompson, M. E.; Forrest, S. R. Nearly 100\% internal phosphorescence efficiency in an organic light-emitting device. Journal of Applied Physics 2001, 90, 5048-5051.

(2) Baldo, M. A.; O’Brien, D. F.; You, Y.; Shoustikov, A.; Sibley, S.; Thompson, M. E.; Forrest, S. R. Highly efficient phosphorescent emission from organic electroluminescent devices. Nature 1998, 395, 151-154.

(3) Endo, A.; Ogasawara, M.; Takahashi, A.; Yokoyama, D.; Kato, Y.; Adachi, C. Thermally Activated Delayed Fluorescence from Sn4+Porphyrin Complexes and Their Application to Organic Light Emitting Diodes A Novel Mechanism for Electroluminescence. Advanced Materials 2009, 21, 4802-4806.

(4) Uoyama, H.; Goushi, K.; Shizu, K.; Nomura, H.; Adachi, C. Highly efficient organic light-emitting diodes from delayed fluorescence. Nature 2012, 492, 234-238.

(5) Zhang, Q.; Li, J.; Shizu, K.; Huang, S.; Hirata, S.; Miyazaki, H.; Adachi, C. Design of efficient thermally activated delayed fluorescence materials for pure blue organic light emitting diodes. Journal of the American Chemical Society 2012, 134, 14706-14709.

(6) Hirata, S.; Sakai, Y.; Masui, K.; Tanaka, H.; Lee, S. Y.; Nomura, H.; Nakamura, N.; Yasumatsu, M.; Nakanotani, H.; Zhang, Q. et al. Highly efficient blue electroluminescence based on thermally activated delayed fluorescence. Nature Materials 2015, 14, $330-336$.

(7) Sato, K.; Shizu, K.; Yoshimura, K.; Kawada, A.; Miyazaki, H.; Adachi, C. Organic luminescent molecule with energetically equivalent singlet and triplet excited states for organic light-emitting diodes. Physical Review Letters 2013, 110, 1-5. 
(8) Nobuyasu, R. S.; Ren, Z.; Griffiths, G. C.; Batsanov, A. S.; Data, P.; Yan, S.; Monkman, A. P.; Bryce, M. R.; Dias, F. B. Rational Design of TADF Polymers Using a Donor-Acceptor Monomer with Enhanced TADF Efficiency Induced by the Energy Alignment of Charge Transfer and Local Triplet Excited States. Advanced Optical Materials 2016, 4, 597-607.

(9) Dias, F. B.; Santos, J.; Graves, D. R.; Data, P.; Nobuyasu, R. S.; Fox, M. A.; Batsanov, A. S.; Palmeira, T.; Berberan-Santos, M. N.; Bryce, M. R. et al. The role of local triplet excited states and D-A relative orientation in thermally activated delayed fluorescence: Photophysics and devices. Advanced Science 2016, 3, 1-10.

(10) Lee, S. Y.; Yasuda, T.; Komiyama, H.; Lee, J.; Adachi, C. Thermally Activated Delayed Fluorescence Polymers for Efficient Solution-Processed Organic Light-Emitting Diodes. Advanced Materials 2016, 28, 4019-4024.

(11) Samanta, P. K.; Kim, D.; Coropceanu, V.; Brédas, J. L. Up-Conversion Intersystem Crossing Rates in Organic Emitters for Thermally Activated Delayed Fluorescence: Impact of the Nature of Singlet vs Triplet Excited States. Journal of the American Chemical Society 2017, 139, 4042-4051.

(12) de Silva, P.; Kim, C. A.; Zhu, T.; Van Voorhis, T. Extracting Design Principles for Efficient Thermally Activated Delayed Fluorescence (TADF) from a Simple Four-State Model. Chemistry of Materials 2019,

(13) Difley, S.; Beljonne, D.; Voorhis, T. V. On the singlet-triplet splitting of geminate electron-hole pairs in organic semiconductors. Journal of the American Chemical Society 2008, 130, 3420-3427.

(14) Eizner, E.; Martínez-Martínez, L. A.; Yuen-Zhou, J.; Kéna-Cohen, S. Inverting Singlet and Triplet Excited States using Strong Light-Matter Coupling. preprint 2019, arXiv:1903.09251. 
(15) Sato, T.; Uejima, M.; Tanaka, K.; Kaji, H.; Adachi, C. A light-emitting mechanism for organic light-emitting diodes: Molecular design for inverted singlet-triplet structure and symmetry-controlled thermally activated delayed fluorescence. Journal of Materials Chemistry C 2015, 3, 870-878.

(16) Di, D.; Romanov, A. S.; Yang, L.; Richter, J. M.; Rivett, J. P. H.; Jones, S.; Thomas, T. H.; Abdi Jalebi, M.; Friend, R. H.; Linnolahti, M. et al. High-performance light-emitting diodes based on carbene-metal-amides. Science 2017, 356, 159-163.

(17) Olivier, Y.; Sancho-Garcia, J. C.; Muccioli, L.; D’Avino, G.; Beljonne, D. Computational Design of Thermally Activated Delayed Fluorescence Materials: The Challenges Ahead. Journal of Physical Chemistry Letters 2018, 9, 6149-6163.

(18) Olivier, Y.; Yurash, B.; Muccioli, L.; Avino, G. D.; Mikhnenko, O.; Adachi, C.; DAvino, G.; Mikhnenko, O.; Sancho-García, J. C.; Adachi, C. et al. Nature of the singlet and triplet excitations mediating thermally activated delayed fluorescence. Physical Review Materials 2017, 075602, 1-6.

(19) Horn, R. A.; Johnson, C. R. Topics in matrix analysis; Cambridge University Press, 1991.

(20) Helgaker, T.; Jørgensen, P.; Olsen, J. Molecular Electronic-Structure Theory; John Wiley \& Sons, Ltd, 2000.

(21) Koch, E. In Correlated Electrons: From Models to Materials; Pavarini, E., Koch, E., Anders, F., Jarrell, M., Eds.; Forschungszentrum Jlich GmbH, Institute for Advanced Simulation, 2012; Chapter 7.

(22) Elliott, P.; Goldson, S.; Canahui, C.; Maitra, N. T. Perspectives on double-excitations in TDDFT. Chemical Physics 2011, 391, $110-119$. 
(23) Dreuw, A.; Head-Gordon, M. Single-Reference ab Initio Methods for the Calculation of Excited States of Large Molecules. Chemical Reviews 2005, 105, 4009-4037.

(24) Moore, B.; Sun, H.; Govind, N.; Kowalski, K.; Autschbach, J. Charge-Transfer Versus Charge-Transfer-Like Excitations Revisited. Journal of Chemical Theory and Computation 2015, 11, 3305-3320.

(25) Maitra, N. T.; Zhang, F.; Cave, R. J.; Burke, K. Double excitations within timedependent density functional theory linear response. The Journal of Chemical Physics 2004, 120, 5932-5937.

(26) Mazur, G.; Wodarczyk, R. Application of the dressed time-dependent density functional theory for the excited states of linear polyenes. Journal of Computational Chemistry 2009, 30, 811-817.

(27) Shao, Y.; Head-Gordon, M.; Krylov, A. I. The spinflip approach within time-dependent density functional theory: Theory and applications to diradicals. The Journal of Chemical Physics 2003, 118, 4807-4818.

(28) Wu, Q.; Van Voorhis, T. Direct optimization method to study constrained systems within density-functional theory. Physical Review A 2005, 72, 024502.

(29) Kaduk, B.; Van Voorhis, T. Communication: Conical intersections using constrained density functional theoryconfiguration interaction. The Journal of Chemical Physics 2010, 133, 061102.

(30) Minezawa, N.; Gordon, M. S. Optimizing Conical Intersections by SpinFlip Density Functional Theory: Application to Ethylene. The Journal of Physical Chemistry A 2009, 113, 12749-12753.

(31) Huix-Rotllant, M.; Natarajan, B.; Ipatov, A.; Muhavini Wawire, C.; Deutsch, T.; Casida, M. E. Assessment of noncollinear spin-flip TammDancoff approximation time- 
dependent density-functional theory for the photochemical ring-opening of oxirane. Phys. Chem. Chem. Phys. 2010, 12, 12811-12825.

(32) Northey, T.; Stacey, J.; Penfold, T. J. The role of solid state solvation on the charge transfer state of a thermally activated delayed fluorescence emitter. Journal of Materials Chemistry C 2017, 5, 11001-11009.

(33) Sun, H.; Hu, Z.; Zhong, C.; Chen, X.; Sun, Z.; Brédas, J. L. Impact of Dielectric Constant on the Singlet-Triplet Gap in Thermally Activated Delayed Fluorescence Materials. Journal of Physical Chemistry Letters 2017, 8, 2393-2398.

(34) Subotnik, J. E. Communication: Configuration interaction singles has a large systematic bias against charge-transfer states. The Journal of Chemical Physics 2011, 135, 071104 .

(35) Liu, X.; Fatehi, S.; Shao, Y.; Veldkamp, B. S.; Subotnik, J. E. Communication: Adjusting charge transfer state energies for configuration interaction singles: Without any parameterization and with minimal cost. The Journal of Chemical Physics 2012, 136, 161101.

(36) Thouless, D. Stability conditions and nuclear rotations in the Hartree-Fock theory. Nuclear Physics 1960, 21, $225-232$.

(37) Leupin, W.; Wirz, J. Low-Lying Electronically Excited States of Cycl[3.3.3]azine, a

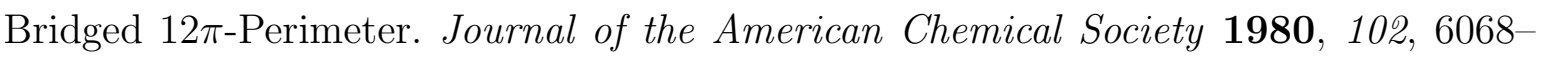
6075 .

(38) Klán, P.; Wirz, J. Photochemistry of Organic Compounds: From Concepts to Practice; John Wiley \& Sons, Ltd, 2009.

(39) Shao, Y.; Gan, Z.; Epifanovsky, E.; Gilbert, A. T.; Wormit, M.; Kussmann, J.; Lange, A. W.; Behn, A.; Deng, J.; Feng, X. et al. Advances in molecular quantum 
chemistry contained in the Q-Chem 4 program package. Molecular Physics 2015, 113, $184-215$.

(40) Head-Gordon, M.; Rico, R. J.; Oumi, M.; Lee, T. J. A doubles correction to electronic excited states from configuration interaction in the space of single substitutions. Chemical Physics Letters 1994, 219, 21 - 29.

(41) Wormit, M.; Rehn, D. R.; Harbach, P. H.; Wenzel, J.; Krauter, C. M.; Epifanovsky, E.; Dreuw, A. Investigating excited electronic states using the algebraic diagrammatic construction (ADC) approach of the polarisation propagator. Molecular Physics 2014, 112, 774-784.

(42) Stanton, J. F.; Bartlett, R. J. The equation of motion coupled-cluster method. A systematic biorthogonal approach to molecular excitation energies, transition probabilities, and excited state properties. The Journal of Chemical Physics 1993, 98, 7029-7039. 